\title{
LEXICOGRAPHIC PARTIAL ORDER
}

BY

\author{
HENRY CRAPO
}

\begin{abstract}
Given a (partially) ordered set $P$ with the descending chain condition, and an ordered set $Q$, the set $Q^{P}$ of functions from $P$ to $Q$ has a natural lexicographic order, given by $f<g$ if and only if $f(y)<g(y)$ for all minimal elements of the set $\{x ; f(x) \neq g(x)\}$ where the functions differ.

We show that if $Q$ is a complete lattice, so also is the set $Q^{P}$, in the lexicographic order. The same holds for the set $\operatorname{Hom}(P, Q)$ of order-preserving functions, and for the set $\mathrm{Op}(P)$ of increasing order-preserving functions on the set $P$.

However, the set $\mathrm{Cl}(P)$ of closure operators on $P$ is not necessarily a lattice even if $P$ is a complete lattice.
\end{abstract}

We prove that the set $Q^{P}$ of functions from a partially ordered set $P$ into a complete lattice $Q$ is a complete lattice in the "ordinal power" or "lexicographic" order if the set $P$ satisfies the descending chain condition. This was a matter of conjecture in Birkhoff's paper [2], where he outlined an extension of cardinal and ordinal arithmetic to partially ordered sets. Some years later, Day [3] pointed out a few errors in the original paper, and studied the question of transitivity of lexicographic orders in great detail. But his description of the lattice operations in an ordinal power was incorrect.

Under the above assumptions on $P$ and $Q$, we prove also that the set $\operatorname{Hom}(P, Q)$ of order-preserving functions from $P$ into $Q$ is a complete lattice in the induced lexicographic order. If $P=Q$, we show that the order operators (increasing, order-preserving functions) form a complete lattice. However, neither closure operators nor geometric closure operators are lattice ordered by the lexicographic order.

We use the abbreviations ordered set for partially-ordered set, order for partial order. A subset $B$ of an ordered set $P$ is an order filter if and only if $x \in B$ and $x \leqslant y$ imply $y \in B$. An order ideal is an order filter in the opposite order. For every order ideal $B$ in an ordered set $P$, define

$$
B^{+}=B \cup\{x ; x \text { is minimal in } P-B\} \text {. }
$$

Recall that if an ordered set $P$ has the descending chain condition (d.c.c.),

Received by the editors September 10, 1972 and October 19, 1972 and, in revised form February 1, 1974 and May 17, 1976.

AMS (MOS) subject classifications (1970). Primary 06A10, 06A23, 06 A15.

Key words and phrases. Partial order, order, lattice, complete lattice, closure operator, lexicographic order. 
then every order filter $A$ in $P$ is generated by its minimal elements, that is:

$$
A=\{y ; \exists x \text { minimal in } A, x<y\} .
$$

Since our primary concern is with the ordinal power, or lexicographic, order on functions, we shall use the notations $<(\mathrm{pt}), \wedge(\mathrm{pt}), \vee(\mathrm{pt})$ for pointwise order, infimum, and supremum.

1. Powers of ordered sets. Given two ordered sets $P$ and $Q$, we impose a natural lexicographic order on the set $Q^{P}$ of functions from the set $P$ into the set $Q$. Given two functions $f, g: P \rightarrow Q$ we want $f<g$ if the strict inequality $f<g$ holds at all minimal elements of the set $\{x \in P ; f(x) \neq g(x)\}$ on which the functions disagree. Of course if $f(x) \neq g(x)$ for all elements $x \in P$ (say $f(x)<g(x)$ for all $x$ ) and if $P$ is an infinite descending sequence, then we may conclude both $f \leqslant g$ and $g \leqslant f$. This reveals an undesirable lack of antisymmetry, and forces a conclusion inconsistent with the pointwise order. Birkhoff noted the lack of antisymmetry. Day observed further that a relation so defined need not be transitive. We are led to assume the descending chain condition for the order on the domain (or "exponent") $P$, and shall do so henceforth.

Having imposed the descending chain condition on the order of the exponent, we have a systematic procedure for making comparisons between functions, and can therefore introduce a partial order on the set $Q^{P}$ of functions from $P$ to $Q$. If $f$ and $g$ are two such functions (not necessarily order-preserving) from $P$ to $Q$, we say $f \leqslant g$ if and only if the strict inequality $f(y)<g(y)$ holds at every minimal element $y$ of the set upon which the functions differ.

Proposition 1. Let $P($ d.c.c. $)$ and $Q$ be ordered sets and let $f, g$ be two functions from the set $P$ into the set $Q$. Each of the following conditions is equivalent to the relation $f \leqslant g$.

(1) $\forall x \in P$ such that $f(x) \neq g(x), \exists y \in P, y \leqslant x$, with $f(y)<g(y)$;

(2) $\forall x \in P$ such that $f(x) \nless g(x), \exists y \in P, y<x$, with $f(y)<g(y)$.

Proof. If two functions $f, g$ satisfy condition (1) and $f(x) \leqslant g(x)$, then $f(x) \neq g(x)$ and there is an element $y \leqslant x$ such that $f(y)<g(y)$. But $f(x)<g(x)$, so $x \neq y$ and $y<x$, as required in condition (2). Assuming condition (2), let $y$ be a minimal element in the set $\{x ; f(x) \neq g(x)\}$. If $f(y)<g(y)$, then $f(y) \leqslant g(y)$ and there exists $z<y$ such that $f(z)<g(z)$. In particular, $f(z) \neq g(z)$, a contradiction. Thus $f(y)<g(y)$, and $f<g$. Assuming, finally, that $f \leqslant g$, let $x$ be an element at which $f(x) \neq g(x)$. The set $K=\{z ; z \leqslant x$ and $f(z) \neq g(z)\}$ is not empty because $x \in K$. Choose a minimal element $y$ of the set $K$. Then $y$ is also minimal in the set $\{z$; $f(z) \neq g(z)\}$, so $f(y)<g(y)$, and since $y \leqslant x$, condition (1) holds. 
Proposition 2 (DAY). The power $Q^{P}$ of ordered sets, where $P$ satisfies the d.c.c., is an ordered set.

Proof. Given two functions $f, g \in Q^{P}$, let $E$ be the order ideal

$$
E=\{x ; \forall y \leqslant x, f(y)=g(y)\} .
$$

If $E$ is a proper subset of $P$, then by the d.c.c. the complementary set $P-E$ has a minimal element $x$. If $f \leqslant g$ and $g \leqslant f$, then $f(x)<g(x)$ and $g(x)<$ $f(x)$, a contradiction. Thus $E=P$ and $f=g$. Conversely, if $f=g$, then $P-E$ is empty, and $f<g$. Thus the relation $<$ is antisymmetric and reflexive.

To prove transitivity, assume $f \leqslant g, g \leqslant h$, and let $x$ be a minimal element such that $f(x) \neq h(x)$. If $f(x)<h(x)$ then $f(x) \leqslant h(x)$, and either $f(x)<$ $g(x)$ or $g(x) \leqslant h(x)$. In either case, the set $\{y ; y<x$ and $f(y) \neq g(y)\}=$ $\{y ; y<x$ and $g(y) \neq h(y)\}$ is nonempty, and has a minimal element $z$. The element $z$ is minimal in both sets $\{y ; f(y) \neq g(y)\}$ and $\{y ; g(y) \neq h(y)\}$. Since $f \leqslant g$ and $g \leqslant h, f(z)<g(z)$ and $g(z)<h(z)$, so $f(z)<h(z)$, contradicting the definition of $x$.

This lexicographic order of $Q^{P}$ is comparable with and stronger than the pointwise order on functions from $P$ to $Q$. If two functions $f, g$ stand in the pointwise order $f \leqslant(\mathrm{pt}) g$, then at every point $x$ where $f(x) \neq g(x)$, we have $f(x)<g(x)$, so $f \leqslant g$ in the lexicographic order.

2. Powers of lattices. If the exponent $P$ satisfies the d.c.c. and the base $Q$ is a complete lattice, then the power $Q^{P}$ is also a complete lattice. Birkhoff and Day provide some assistance in defining the lattice operations supremum and infimum. Birkhoff's recursive definition [2, p. 295, lines 18-23] requires both chain conditions: d.c.c. for the existence of minimal elements generating order filters in $P$, and a.c.c. so that the recursive definition reaches all elements of $P$. He proceeds as follows. If the infimum $f=\wedge_{i \in I} f_{i}$ has been defined on an order ideal $A \subseteq P$, then for each minimal element $y$ of the complementary order filter $P-A$ we define a set $I_{y}$ of indices

$$
I_{y}=\left\{i \in I \text {; for no } z<y \text { is } f(z)<f_{i}(z)\right\}
$$

and define

$$
f(y)=\bigwedge_{i \in \lambda_{y}} f_{i}(y)
$$

The idea here is that if the relation $f<f_{i}$ is not already established at some point $z<y$, it must not be negated at $y$. Definitions (3), (4) are necessary and sufficient to that end. Birkhoff's definition assigns a value $f(y)$ only for those elements $y$ in the order ideal $A \subseteq P$ where

$$
A_{0}=\varnothing, \quad A_{i}=\left(A_{i-1}\right)^{+} \text {for } 1,2, \ldots, \quad \text { and } A=\bigcup_{i=0}^{\infty} A_{i}
$$


The order ideal $A$ is equal to $P$ if and only if for every element $y \in P$, the order ideal $\{x ; x<y\}$ satisfies the ascending chain condition.

Day's definition [3, p. 35, lines 22-31] requires only the descending chain condition, but is incorrect. After an appropriate translation into our present notation (Day defined the power in terms of the opposite order on the exponent), we outline his procedure as follows. Given a set $\left\{f_{i} ; i \in I\right\}$ of functions $f_{i}: P \rightarrow Q$, let $F_{i}$ be the order filter

$$
F_{i}=\left\{x \in P ; \exists y \leqslant x, \bigwedge_{j} f_{j}(y)<f_{i}(y)\right\} .
$$

Let $E_{i}$ be the complementary order ideal $E_{i}=P-F_{i}$, and let $G=\cap_{i} E_{i}$. Day claims that the infimum coincides with the pointwise infimum on the set $G^{+}$, and otherwise has value 1 .

EXAMPLE 1. For a simple counterexample, take $f_{1} \equiv 0, f_{2}(x)=1-x$ in the power $C^{C}$ where $C$ is the two-element chain of integers $\{0,1\}$ with $0<1$. Then $G=C-F_{2}=\varnothing$ and $G^{+}=\{0\}$, so Day's definition for the infimum $f$ yields $f(0)=0 \wedge 1=0, f(1)=1$, and $f \ll f_{1}$.

Example 2 will show that the infimum cannot always take the value 1 even on the complement of the larger order ideal $\left(\cup_{i} E_{i}\right)^{+}$.

EXAMPLE 2. Let $B=\{0, a, b, 1\}$ be the Boolean algebra with $0<a<1$, $0<b<1$. Let $f_{1}$ take values $a, a, b, b$ and let $f_{2}$ take values $1, b, a, a$ on $0, a, b, 1$, respectively. Then $f_{1}<f_{2}$, so $f_{1} \wedge f_{2}$ must equal $f_{1}$ and $\left(f_{1} \wedge f_{2}\right)(1)=b$, but $\left(\cup_{i} E_{i}\right)^{+}=E_{1}^{+}=\{0, a, b\}$.

We give a correct definition below, lines (7-9). Our definition of the lattice operations uses the power order on the restrictions of functions $f: P \rightarrow Q$ to principal order ideals

$$
P_{x}=\{y \in P ; y \leqslant x\},
$$

for various elements $x \in P$. By " $f \leqslant g$ on $P_{x}$ " we mean $\left.f\right|_{P_{x}} \leqslant\left. g\right|_{P_{x}}$ in $Q^{P_{x}}$. The following proposition, concerning the power order on restrictions to principal ideals, will make possible a substantial simplification of later proofs.

Proposition 3. The set $\left\{x, " f \leqslant g\right.$ on $P_{x}$ " $\}$ is an order ideal in the set $P$. The minimal elements of the complementary order filter are precisely those elements $x$ such that

$$
f(x) \leqslant g(x) \text { but } f(y)=g(y) \text { for all } y<x .
$$

Proof. The statement " $f \leqslant g$ on $P_{x}$ " holds if and only if a certain statement

$$
\text { (namely: } f(y) \leqslant g(y) \Rightarrow \exists z<y, f(z)<g(z) \text { ) }
$$

holds for all elements $y \leqslant x$. Thus $\left\{x\right.$; " $f \leqslant g$ on $P_{x}$ " $\}$ is an order ideal.

If $x$ is an element for which line (6) applies, then " $f=g$ on $P_{y}$ " for all 
$y<x$, but " $f<g$ on $P_{x}$ ", so $x$ is a minimal element of the complementary order filter. Conversely, if " $f \leqslant g$ on $P_{x}$ " is false, but " $f<g$ on $P_{y}$ " is true for all elements $y<x$, the statement $f(s) \leqslant g(s) \Rightarrow \exists t<s, f(t)<g(t)$ holds for all $s<x$ and must fail at $s=x$. That is: $f(x) \leqslant g(x)$ and for all $y<x$, $f(y)<g(y)$. But if $f(y) \neq g(y)$ for some $y<x$, then $f(z)<g(z)$ for some $z \leqslant y$ and therefore for some $z<x$, a contradiction. Thus $f(y)=g(y)$ for all $y<x$, and condition (6) holds.

We now define lattice operations on the power $Q^{P}$ if $Q$ is a complete lattice and $P$ has the d.c.c. Given a set $\left\{f_{i} ; i \in I\right\}$ of functions in $Q^{P}$, then for each index $i \in I$ define an order ideal

$$
E_{i}=\left\{x ;(\forall j \in I) f_{i} \leqslant f_{j} \text { on } P_{x}\right\}
$$

For each element $x \in P$ define a set of indices

$$
A_{x}=\left\{i \in I ; x \in E_{i}^{+}\right\} \text {. }
$$

Then the infimum $\wedge_{i} f_{i}$ is given by the expression

$$
\left(\bigwedge_{i} f_{i}\right)(x)=\bigwedge_{i \in A_{x}} f_{i}(x)
$$

The supremum is the infimum with respect to the opposite order on the lattice $Q$, the original order on the exponent $P$. Thus we use the order ideals

$$
F_{i}=\left\{x ;(\forall j \in I) f_{i}>f_{j} \text { on } P_{x}\right\}
$$

we let

$$
A_{x}=\left\{i \in I ; x \in F_{i}^{+}\right\}
$$

and set

$$
\left(\bigvee_{i} f_{i}\right)(x)=\bigvee_{i \in A_{x}} f_{i}(x)
$$

THEOREM 1. If $Q$ is a complete lattice and $P$ is an ordered set with d.c.c., the operations $\wedge$ and $\bigvee$ defined above are the complete lattice operations on the power $Q^{P}$.

Proof. Let $f=\bigwedge_{i} f_{i}$ as defined in equation (9). Assume that for some element $x \in P, f(x) \leqslant f_{i}(x)$. By equation (9), $i \notin A_{x}$, and by equation (8), $x \notin E_{i}^{+}$, so there is an element $y<x, y$ minimal in $P-E_{i}$. For some function $f_{j}, f_{i} \leqslant f_{j}$ on $P_{y}$. Since $y$ is a minimal such element, Proposition 3 yields $f_{i}(z)=f_{j}(z)$ for all $z<y, y \in E_{j}^{+}, f_{i}(y) \& f_{j}(y)$, and

$$
f(y) \leqslant f_{i}(y) \wedge f_{j}(y)<f_{i}(y),
$$

as required. $f$ is a lower bound for all the $f_{i}$.

To show that $f$ is the infimum of the functions $f_{i}$, we show that if a function $g$ is not beneath $f$, it is not a lower bound for all the $f_{i}$. If $g \leqslant f=\wedge_{i} f_{i}$ on $P$, choose a minimal element $x$ such that " $g \leqslant f$ on $P_{x}$ " is false. Then $g(x) \leqslant$ 
$f(x)$ and $g(y)=f(y)$ for all $y<x$, by Proposition 3. Therefore, by equation (9), there exists an index $i \in A_{x}$ such that $g(x) \& f_{i}(x)$. Since $i \in A_{x}$, we know $x \in E_{i}{ }^{+}$. For all $y<x, y \in E_{i}$, so

so $g \leqslant f_{i}$.

$$
f_{i}(y)=f(y)=g(y),
$$

We shall use the following proposition to establish a complete lattice order on increasing functions (see $\$ 4$ ).

Proposition 4. For any function $g: P \rightarrow Q$, let $D=\{f: g<(\mathrm{pt}) f\}$ be the set of functions greater than or equal to $g$ in the pointwise order. Then $D$ is a complete lattice in the order induced upon it as a subset of the power $Q^{P}$.

Proof. Assume $f_{i} \in D$, for all $i \in I$. Then for any element $x \in P$, equation (9) expresses $\left(\bigwedge_{i} f_{i}\right)(x)$ as an infimum of function values $f_{i}(x)$, all of which satisfy $g(x) \leqslant f_{i}(x)$. Therefore, $\bigwedge_{i} f_{i} \in D, D$ is a closure system, and $D$ is a complete lattice in the induced lexicographic order.

3. Order-preserving functions. The set $\operatorname{Hom}(P, Q)$ of order-preserving functions from $P$ to $Q$ has a lexicographic order induced by that on the power $Q^{P}$. We prove that under the conditions previously assumed for $P$ and $Q$, $\operatorname{Hom}(P, Q)$ is also a complete lattice.

THEOREM 2. If $Q$ is a complete lattice and $P$ has the d.c.c., then $\operatorname{Hom}(P, Q)$ $\subseteq Q^{P}$ is closed with respect to the infimum in $Q^{P}$, and is therefore a complete lattice in the induced order.

Proof. Assume that functions $f_{i}, i \in I$, are order-preserving, and define subsets $E_{i}, A_{x}$, and the infimum $f=\bigwedge_{i} f_{i}$ as in formulas (7), (8), (9). For every index $i, E_{i}^{+}$is an order ideal. So if $x \leqslant y, A_{x} \supseteq A_{y}$. Thus $f(x)$ is an infimum of a larger set of smaller values: $i \in A_{y} \Rightarrow i \in A_{x}$ and $f_{i}(x)<f_{i}(y)$. Therefore $f(x) \leqslant f(y)$, and $f$ is order-preserving.

The supremum in $Q^{P}$ of order-preserving functions need not be order-preserving, so $\operatorname{Hom}(P, Q)$ is not necessarily a complete sublattice of the power. The following example illustrates this fact.

Example 3. Let $P=Q$ be the Boolean algebra $\{0, a, b, 1\}$ with $0<a<1$ and $0<b<1$. Let $f_{1}$ be the identity function and let $f_{2}$ be the lattice isomorphism which interchanges the elements $a$ and $b$. The supremum $f_{1} \vee f_{2}$ maps $0, a, b, 1$ to $0,1,1,0$, respectively, and is not order-preserving.

For any function $f: P \rightarrow Q$, consider the order-preserving functions $g$ such that $f \leqslant g$. Since $\operatorname{Hom}(P, Q$,$) is a closure system in the power Q^{P}$ there is a least such function, which we denote $f_{0}$.

Proposition 5. Given a function $f \in Q^{P}$, define the order ideal $F=\{x ; f$ is order-preserving on $\left.P_{x}\right\}$ where $P_{x}=\{y ; y \leqslant x\}$. Then 


$$
f_{0}(x)=\underset{y ; y<x, y \in F^{+}}{\bigvee} f(y) .
$$

Proof. The function $f_{0}$ defined by (10) is order-preserving, because if $x \leqslant y,\left\{z ; z \leqslant x, z \in F^{+}\right\} \subseteq\left\{z ; z \leqslant y, z \in F^{+}\right\}$. We prove that $f \leqslant f_{0}$. Assume $f(x) \ll f_{0}(x)$ for some element $x \in P$. Then $x \notin F^{+}$, so let $y$ be a minimal element such that $y<x, y \notin F$. The function $f$ is order-preserving on all order ideals $P_{z}$ for $z<y$, but is not order-preserving on $P_{y}$. So there is an element $z<y$ such that $f(z) \& f(y)$. Since $z \in F$,

$$
f_{0}(y) \geqslant f(z) \bigvee f(y)>f(y) .
$$

Thus $f \leqslant f_{0}$.

Let $g$ be an order-preserving function, and assume $f \leqslant g$. We prove $f_{0} \leqslant g$. Assume that for some element $x, f_{0}(x) \ll g(x)$. By equation (10), there is an element $y \in F^{+}, y \leqslant x$, such that $f(y) \leqslant g(x)$, so certainly $f(y) \leqslant g(y)$. Since $f \leqslant g$, there is an element $z<y$ such that $f(z)<g(z)$. We know $z \in F$, so $f_{0}(z)=f(z)$, and $f_{0}(z)<g(z)$ as required.

COROLlary. The lattice operations in $\operatorname{Hom}(P, Q)$ are $\wedge$ and $(\bigvee)_{0}$.

4. Order operators. A function $f: P \rightarrow P$ defined on an ordered set $P$ is increasing if and only if $(\forall x \in P) x \leqslant f(x)$. Assume $P$ is a complete lattice with d.c.c., and consider the increasing functions on $P$ as a subset of the power $P^{P}$.

THEOREM 3. If $P$ is a complete lattice with d.c.c., then the increasing functions on $P$ form a complete lattice in the induced order.

Proof. A function $f$ is increasing if and only if $e \leqslant(\mathrm{pt}) f$, where $e$ is the identity function on $P$. By Proposition 4, the increasing functions form a closure system in the power $P^{P}$.

For any function $f \in P^{p}$, let $f_{\uparrow}$ be the least increasing function which is greater than or equal to $f$ in the power order. Caution: it is not necessarily true that $f \leqslant(\mathrm{pt}) f_{\uparrow}$, so $f_{\uparrow}$ cannot be computed as the pointwise supremum $f \vee(\mathrm{pt}) e$. Furthermore, the lexicographic supremum $f \vee e$ need not be increasing, so it also does not provide a construction of $f_{\uparrow}$.

Example 4. Let $C_{4}=\{0,1,2,3\}$ be the four-element chain. Let the function $f$ take values $1,0,3,3$ on $0,1,2,3$, respectively. Then $f_{\uparrow}$ takes values $1,1,2,3$, while $f \geqslant e$, so the supremum $f \vee e=f$ has values $1,0,3,3$ and the pointwise supremum $f \vee(\mathrm{pt}) e$ has values $1,1,3,3$.

Proposition 6. For any function $f: P \rightarrow P$ on a complete lattice $P$ with d.c.c., the infimum $f_{\uparrow}$ of all increasing functions above $f$ is given by the formulas: 


$$
\begin{aligned}
G & =\{x ;(\forall y \leqslant x) y \leqslant f(y)\}, \\
f_{\uparrow}(x) & =x \vee f(x) \text { for } x \in G^{+}, \\
& =x \text { for } x \notin G^{+} .
\end{aligned}
$$

Proof. The function $f_{\uparrow}$ defined by equations (11) is increasing. If, for some $x \in P, f(x) \leqslant f_{\uparrow}(x)$, then $x \notin G^{+}$, so there exists an element $y<x, y$ minimal in $P-G$. Then $y \leqslant f(y)$, so $f(y)<y \vee f(y)=f_{\uparrow}(y)$, and $f<f_{\uparrow}$.

Assume $f \leqslant g$ for some increasing function $g$, and let $x$ be an element such that $f_{\uparrow}(x) \leqslant g(x)$. Then $x \in G^{+}$, for otherwise $f_{\uparrow}(x)=x \leqslant g(x)$. Thus $f(x) \leqslant g(x)$. Since $f \leqslant g$, there exists an element $y<x$ such that $f(y)<$ $g(y)$. But $y \in G$, so $y \leqslant f(y)=f_{\uparrow}(y)$, and $f_{\uparrow}(y)<g(y)$, and $f_{\uparrow} \leqslant g$, as required.

An order operator on an ordered set $P$ is a function $f: P \rightarrow P$ which is both increasing and order-preserving. Denote by $\mathrm{Op}(P)$ the set of order operators on $P$. As an immediate consequence of Theorems 2 and 3 we have

THEOREM 4. If $P$ is a complete lattice with d.c.c., then the set $\mathrm{Op}(P)$ of order operators on $P$ is a complete lattice in the induced order.

The supremum in $P^{P}$ of increasing functions need not be increasing. In fact, the supremum in $P^{P}$ of order operators need be neither increasing nor order-preserving.

EXAMPLE 5. On the Boolean algebra $\{0, a, b, 1\}$ with $0<a<1,0<b<1$, let $f_{1}(x)=x \vee a$ and $f_{2}(x)=x \vee b$. Then, as defined in line $\left(7^{*}\right), F=\varnothing, F^{+}$ $=\{0\}$, so $\left(f_{1} \vee f_{2}\right)=a \vee b=1$ and $\left(f_{1} \vee f_{2}\right)(x)=0$ for $x \neq 0$.

For any function $f: P \rightarrow P$, let $f_{*}$ denote the infimum of all order operators greater than or equal to $f$ in the power $P^{P}$. If any two consecutive functions are equal in the sequence $f \leqslant f_{0} \leqslant f_{0 \uparrow} \leqslant f_{0 \uparrow 0} \leqslant f_{0 \uparrow 0 \uparrow} \cdots$, then all subsequent functions in the sequence are equal, and equal to $f_{*}$. The operators 0 and $\uparrow$ do not commute, as the following examples show. If $f$ is order-preserving, $f_{\uparrow}$ need not be order-preserving. If $f$ is increasing, $f_{0}$ need not be increasing. In fact, iteration of the operators 0 and $\uparrow$ can produce an infinite sequence of distinct functions.

EXAMPLE 6. Let $T$ be the Boolean algebra of subsets of the set $\{a, b, c\}$. Let $f(\varnothing)=\varnothing$, let $f$ cyclically permute the one-element subsets, and otherwise take value $1=\{a, b, c\}$. Then $f$ is order-preserving, and is increasing only on the set $G=\{\varnothing\}$ as defined in equation (11). $G^{+}=\{\varnothing, a, b, c\}$, and $f_{\uparrow}$, as indicated in Figure 1, is not order-preserving.

EXAMPLE 7. Let $C_{4}$ be the 4 element chain $0<1<2<3$. Let $f(0)=2$ and otherwise $f(x)=x$. The order ideal $F$ defined in Proposition 5 is the set $\{0\}$, and $F^{+}=\{0,1\}$, so $f_{0}(3)=2 \vee 1=2$, and $f_{0}$ is not increasing.

If $P$ is a chain, as in Example 7, iteration of the operators 0 and $\uparrow$ quickly 
produces an order operator. This phenomenon is clearly shown in Figure 2. Here we plot a function $f: R^{+} \rightarrow R^{+}$on the nonnegative real number line $R^{+}$(ignoring for a moment the fact that the d.c.c. does not hold).
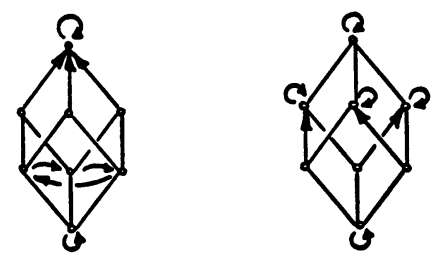

Figure 1

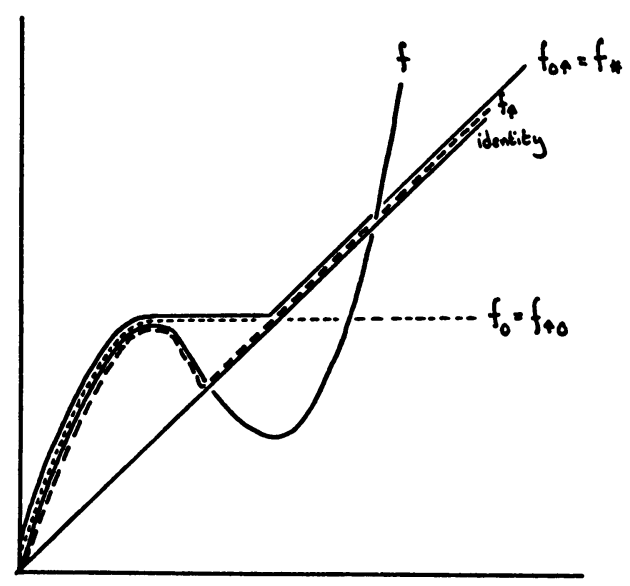

FIGURE 2

Proposition 7. If $P$ is a chain (with d.c.c.) then for any function $f: P \rightarrow P$, $f_{0 \uparrow}$ is order-preserving, so $f_{0 \uparrow}=f_{*}$.

Proof. Let $g=f_{0}$, and assume $x<y$ in $P$. Since $g$ is order-preserving, if $g_{\uparrow}(x)<g_{\uparrow}(y)$, we must have $g_{\uparrow}(x)=x \vee g(x), g$ is increasing at all elements $s<x, g_{\uparrow}(y)=y, g$ is not increasing at some element $t<y$, and $y<x \vee$ $g(x)$, so $y<g(x)$, because $P$ is a chain. Since $g$ is increasing beneath $x$, $t<x$, so $x<t$. But $g(t)<t<y<g(x)$, contradicting the assumption that $g$ is order-preserving.

Example 8 shows that iteration of the operations 0 and $\uparrow$ may produce an infinite sequence of distinct functions.

Example 8. Let $P$ be the distributive lattice $\mathbf{Z}_{2} \times \mathbf{Z}^{+}$, the product of a two-element chain $\mathbf{Z}_{2}=\{0,1\}$ with the positive integers $\mathbf{Z}^{+}$, linearly ordered. Let us use the notation $i$ for the pair $(0, i)$ and $i^{\prime}$ for the pair $(1, i)$. Let $f$ be the constant function with value $1^{\prime}$. Then $f=f_{0}$, and we obtain by applying $n$th 


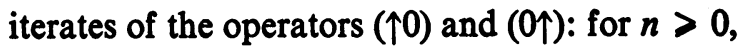

$$
f_{(\uparrow 0)^{n}}=f_{0(\uparrow 0)}:: \begin{cases}i, i^{\prime} \rightarrow i^{\prime} & \text { for } i<2 n+1, \\ i, i^{\prime} \rightarrow(2 n+1)^{\prime} & \text { otherwise, }\end{cases}
$$

and for $n>0$,

$$
f_{(0 \uparrow)^{n}}=f_{\uparrow(0 \uparrow)^{n-1}}: \begin{cases}i, i^{\prime} \rightarrow i^{\prime} & \text { for } i<2 n, \\ i \rightarrow i & \\ i^{\prime} \rightarrow i^{\prime} & \text { otherwise. }\end{cases}
$$

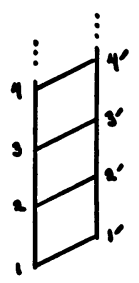

P
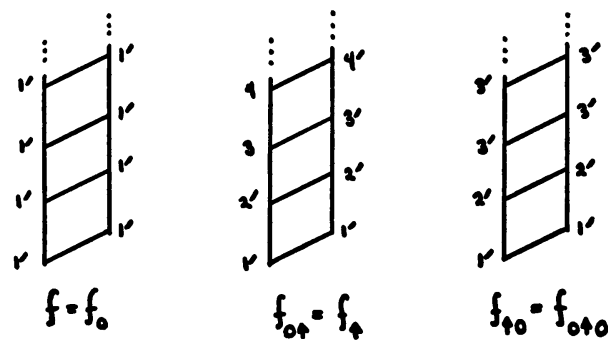

$f=f_{0}$

$f_{40}=f_{040}$

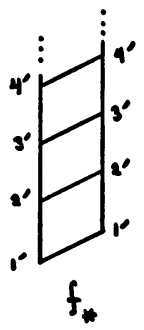

FIGURE 3

Proof. Setting $n=0$ in the first formula, we find $f_{(\uparrow 0)^{\circ}}=f$. For $f$ itself, and for any iterate in which 0 was the last operator used, say $g=f_{(\uparrow))^{n} \text {, we show }}$

$$
g_{\uparrow}=f_{(\uparrow 0)_{\uparrow}^{n}}=f_{\uparrow(0 \uparrow)^{n}}=f_{(0 \uparrow)^{n+1}} \text {, }
$$

agreeing with the definition given in the second formula. The function $g$ is increasing for all $x<(2 n+1)^{\prime}$, and fails to be increasing at $g(2 n+2)=(2 n$ $+1)^{\prime}$. Thus 


$$
g_{\uparrow}:\left\{\begin{array}{l}
i, i^{\prime} \rightarrow i^{\prime} \text { for } i \leqslant 2 n+1, \\
2 n+1 \rightarrow(2 n+2) \vee(2 n+1)^{\prime}=(2 n+2)^{\prime}, \\
i \rightarrow i \text { for } i \geqslant 2 n+3, \\
i^{\prime} \rightarrow i^{\prime} \text { for } i \geqslant 2 n+2,
\end{array}\right.
$$

which agrees with the definition of $f_{(0 \uparrow)^{n+1}}$.

For any iterate in which $\uparrow$ was the last operator used, to say $g=f_{(0 \uparrow)^{n}}$ $(n \geqslant 1)$, we show

$$
g_{0}=f_{(0 \uparrow)^{n} 0}=f_{0(\uparrow 0)^{n}}=f_{(\uparrow 0)^{n}}
$$

as defined in the first formula. The function $g$ is order-preserving for $x<(2 n)^{\prime}$, but fails at $g(2 n+1)=2 n+1 \forall g(2 n)=(2 n)^{\prime}$. Thus

$$
g_{0}:\left\{\begin{array}{l}
i, i^{\prime} \rightarrow i^{\prime} \text { for } i \leqslant 2 n, \\
i, i^{\prime} \rightarrow g(2 n) \vee g(2 n+1)=(2 n)^{\prime} \vee(2 n+1)=(2 n+1)^{\prime} \\
\text { for } i \geqslant 2 n+1,
\end{array}\right.
$$

which agrees with the definition of $f_{(\uparrow 0)^{n}}$ given in the first formula.

Proposition 8. For any function $f: P \rightarrow P$ on a complete lattice $P$ with d.c.c., the infinum $f_{*}$ of all order operators above $f$ is given by the formulas:

$$
\begin{aligned}
H & =\left\{x ; f \text { is an order operator on } P_{x}\right\}, \\
f_{*}(x) & =x \bigvee_{y ; y<x, y \in H^{+}} f(y)
\end{aligned}
$$

where $P_{x}$ is the principal order ideal $\{y ; y<x\}$.

Proof. $f_{*}$, as defined by equations (12), is increasing, and is order-preserving because $x<y$ implies

$$
\left\{z ; z \leqslant x, z \in H^{+}\right\} \subseteq\left\{z ; z<y, z \in H^{+}\right\} .
$$

Assume that $g$ is an order operator, with $f<g$. We prove that $f_{*}<g$. If for some $x \in P, f_{*}(x) \leqslant g(x)$, then

$$
x \bigvee_{y ; y<x, y \in H^{+}} f(y)<g(x)
$$

Since $x<g(x)$, there must exist an element $y<x, y \in H^{+}$, such that $f(y)<g(x)$. Now $g(y) \leqslant g(x)$, so $f(y) \leqslant g(y)$. We have assumed that $f<g$, so there exists an element $z<y$ with $f(z)<g(z)$. But $z \in H$, so $f_{*}(z)<g(z)$, as required.

COROLlaRY. The lattice operations in $\mathrm{Op}(P)$ are $\wedge$ and $(\mathrm{V})_{*}$.

5. Closure operators. By a closure operator (or closure) on an ordered set $P$ we mean a function $f: P \rightarrow P$ which is increasing, order-preserving, and idempotent. We denote by $C(P)$ the set of closures on the ordered set $P$. We have already seen in Example 5 two closure operators whose supremum in $P^{P}$ is neither increasing, order-preserving, nor idempotent. It is still conceivable, 
however, that $C(P)$ could be closed with respect to either suprema or infima in the lattice $\operatorname{Op}(P)$. This is not the case. The next example shows two closure operators such that neither their infimum nor their supremum in $O p(P)$ is idempotent.

EXAMPLE 9. Let $T$ be the Boolean algebra in Example 6. We indicate closures $f_{1}$ and $f_{2}$ in Figure 4 by circling the closed elements.

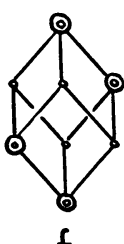

$f_{1}$

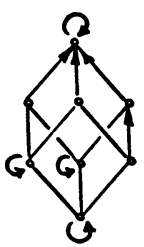

$f_{1} \wedge f_{2}$
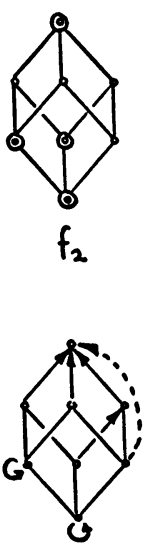

$\left(f_{1} \vee f_{2}\right)_{*}$

FIGURE 4

Actually, we can prove that the set $C(P)$ is not, in general, lattice-ordered. To do this, we must produce an example of four closure operators $f_{1}, f_{2}, g_{1}, g_{2}$ such that $f_{i}<g_{j}$ for all $i, j$, and such that there is no closure operator $h$ such that $f_{i}<h<g_{j}$ for all $i, j$. Such closures are exhibited in Example 10, so we prove

THEOREM 5. The set $C(P)$ of closures on a complete lattice $P$ with d.c.c. is not necessarily lattice ordered.

EXAMPLE 10. Let $Q$ be the Boolean algebra of all subsets of a 4-element set $\{a, b, c, d\}$. We define closure operators by giving their closed elements:

$f_{1}: \varnothing, a, b, c, d, a b, a b d, a b c d$,

$f_{2}: \varnothing, a, b, c, d, b d, a b c, a b c d$,

$\mathrm{g}_{1}: \varnothing, b, d, a b, c d, a b d, a b c d$,

$g_{2}: \varnothing, a, b, b c, b d, a b c, b c d, a b c d$.

Then $\left(f_{1} \vee f_{2}\right)_{*}=g_{1} \wedge g_{2}$ is the order operator $j$ (not a closure) which leaves $\varnothing$ and all one-element sets fixed, maps $a b$ to $a b c$, maps $b d$ to $a b d$, and sends all other sets to $a b c d$. 
6. Geometric closures. A closure $f$ on a Boolean algebra $B(X)$ of all subsets of a set $X$ is geometric if it has the exchange property (13) and finite basis property (14).

$$
\begin{gathered}
\forall A \subseteq X, \forall p, q \in X, p \notin f(A), p \in f(A \cup q) \text { imply } q \in f(A \cup p) . \\
\forall A \subseteq X, \exists C \subseteq A, C \text { finite, } f(C)=f(A) .
\end{gathered}
$$

For the examples we need, it will suffice to describe certain finite sets $X$ of points in affine space, with the understanding that the closure of a set $A \subseteq X$ of points is the set of points in $X$ on the affine subspace generated by $A$.
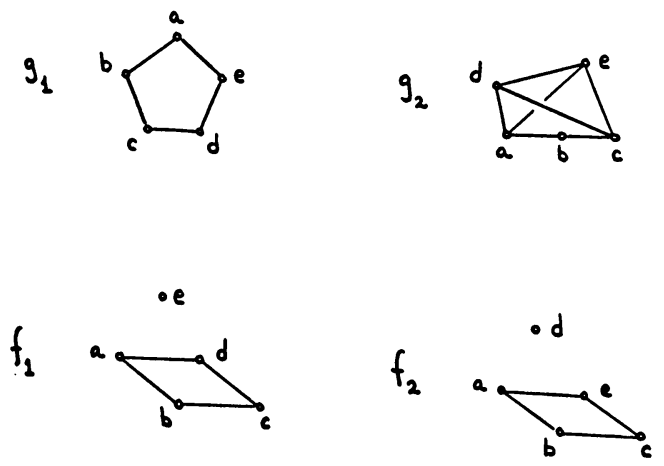

FIGURE 5

EXAMPLE 11. Let $f_{1}, f_{2}, g_{1}, g_{2}$ be the closure operators associated with the geometries indicated in Figure 5. Then $g_{1} \wedge g_{2}$ is a closure operator (not geometric) with closed sets: all subsets with no more than 2 elements, ade, $b d e, c d e$, and abcde. The supremum $\left(f_{1} \vee f_{2}\right)_{*}$ is an order operator which agrees with $g_{1} \wedge g_{2}$ except in that

$$
a b d, a c d, b c d \rightarrow a b c d, \quad a b e, a c e, b c e \rightarrow a b c e .
$$

The only closure operator $h$ satisfying $\left(f_{1} \vee f_{2}\right)_{*} \leqslant h \leqslant g_{1} \wedge g_{2}$ is the closure $g_{1} \wedge g_{2}$, so there is no intermediate geometric closure operator.

THEOREM 6. The set of geometric closures on a finite Boolean algebra $B=B(X)$ is not lattice ordered as a subset of the power $B^{B}$ if $|X| \geqslant 5$.

Proof. If $|X| \geqslant 5$, an example analogous to Example 11 is produced by adjoining $|X|-5$ points by direct sum (each added point is in a new geometric dimension).

There is, however, a closely related lattice order $\leqslant(h)$ on geometries, introduced by Higgs [4]. He defined this order $\leqslant(h)$ as follows. For any geometry $G$ on a set $X$, let $G_{k}$ be the set of subsets of $X$ which have rank $k$ in $G$. Given two geometries $G$ and $H$ on the same set $X$ of points, Higgs sets $G<(h) H$ if and only if $G_{k} \subset H_{k}$ where $k$ is the least rank $i$ for which 
$G_{i} \neq H_{i}$. (This is actually the order opposite to that given by Higgs.) A short computation will verify that if two geometries $G, H$ on a set $X$ have closure operators $g, h$ in the relation $g \leqslant h$ (power order), then $G \leqslant(h) H$ in the Higgs lexicographic order.

7. Dropping the d.c.c. If we are content to compare order-preserving functions in terms of their values on "small" elements only, and if the functions are determined by their values on those "small" elements, we can drop the chain condition, yet obtain a lattice order on the portion of $\operatorname{Hom}(P, Q)$, where $P$ and $Q$ are complete lattices.

Given a complete lattice $P$, define

$$
\begin{gathered}
F_{1}=\{0\}, \quad F_{i}=F_{i-1}+\text { for } i=2,3, \ldots, \\
F=\bigcup_{i} F_{i}, \quad \text { and } \quad P_{F}=F \cup\{1\},
\end{gathered}
$$

where $P_{F} \subseteq P$ has the induced order.

Proposition 9. If $P$ is a complete lattice, $P_{F}$ is a complete lattice with d.c.c.

Proof. $F_{1} \subseteq F_{2} \subseteq \cdots$ is a nested sequence of order ideals, so $F$ is an order ideal and $P_{F} \subseteq P$ is a closure system. Thus $P_{F}$, in the induced order, is a complete lattice. For any subset $A \subseteq P_{F}$, either $A=\varnothing, A=\{1\}$, or there is a least integer $i$ such that $A \cap F_{i} \neq \varnothing$. Let $x$ be an element in $A \cap F_{i}$. Then $x \notin F_{i-1}$, so $x$ is a minimal element of the complement $P-F_{i-1}$. For all $y<x, y \in F_{i-1}$. Since $A \cap F_{i-1}=\varnothing, y \notin A$, and $x$ is minimal in $A$. Thus $P_{F}$ has the d.c.c.

If $P$ and $Q$ are complete lattices, a function $f: P \rightarrow Q$ is finitely defined if and only if for all $x \in P$,

$$
f(x)=\bigvee_{y ; y<x, y \in F} f(y)
$$

Note that every finitely defined function $f: P \rightarrow Q$ determines and is determined by its restriction $f_{F}: P_{F} \rightarrow Q$. Also, both $f_{F}$ and $f$ are order-preserving. We denote by $\operatorname{Hom}_{F D}(P, Q)$ the set of finitely-defined functions $f$ : $P \rightarrow Q$, with power order determined by

$$
f<g \text { if and only if } f_{F}<g_{F} \text { in } Q^{P_{F}} \text {. }
$$

Corollary to Theorem 2. $\operatorname{Hom}_{F D}(P, Q)$ is a complete lattice, for any complete lattices $P, Q$.

\section{BIBLIOGRAPHY}

1. Garrett Birkhoff, An extended arithmetic, Duke Math. J. 3 (1937), 311-316.

2. __ Generalized arithmetic, Duke Math. J. 9 (1942), 283-302. MR 4, 74.

3. Mahlon Day, Arithmetic of ordered systems, Trans. Amer. Math. Soc. 58 (1945), 1-43. MR 7, 1. 
4. Denis Higgs, A lattice order for the set of all matroids on a set, Abstract, Ontario Math. Meeting, Nov. 5, 1966.

Department of Pure Mathematics, University of Waterloo, Watbrloo, Ontarlo, Canada

Current address: Groupe de Recherche: Topologie Structurale, Faculté de l'Amenagement, Université de Montréal, Montréal, Québec, Canada 